\title{
A Planar Cubic Derived from the Logarithm of the Dedekind $\eta$-Function
}

\section{A. LÜTKEN}

$7 / 7$

e first briefly recall the Weierstrass theory of elliptic functions. Every elliptic curve is isomorphic to a Weierstrass cubic

$$
(d \wp / d z)^{2}=4 \wp^{3}-g_{2} \wp-g_{3} \subset \mathbb{C P}_{2}[\wp, d \wp / d z],
$$

where

$$
\wp(z ; \tau)=\frac{1}{z^{2}}+\sum_{n+m \tau \neq 0}\left\{\frac{1}{(z+m+n \tau)^{2}}-\frac{1}{(m+n \tau)^{2}}\right\}
$$

is the meromorphic Weierstrass function with double poles, $z \in \mathbb{C}$ is a point on the curve (a torus), and $\tau \in \mathbb{C}$ is its modulus ("shape"); see Figures 1 and $2 .{ }^{1}$ The coefficients are Eisenstein functions, which come in three varieties that differ only by how they are normalized:

$$
\begin{aligned}
E_{w}(\tau) & =1-\frac{2 w}{B_{w}} \sum_{n=1}^{\infty} \frac{n^{w-1} q^{n}}{1-q^{n}}, \\
G_{w}(\tau) & =2 \zeta(w) E_{w}(\tau), \\
g_{w / 2}(\tau) & =4\left(w^{2}-1\right) G_{w}(\tau),
\end{aligned}
$$

where $2 \leq w \in 2 \mathbb{Z}, q=\exp (2 \pi i \tau), \quad \zeta$ is Riemann's zeta function, and $B_{2}=1 / 6, B_{4}=-1 / 30, B_{6}=1 / 42$, are Bernoulli numbers.
Translations $T(\tau)=\tau+1$ and inversions $S(\tau)=-1 / \tau$ generate the modular group $\Gamma=\operatorname{PSL}(2, \mathbb{Z})$, which is the set of all Möbius transformations with integer coefficients and unit determinant. All $G_{w}$ with $w \geq 4$ are modular forms of weight $w$, which means that they transform like tensors,

$$
G_{w}\left(\frac{a \tau+b}{c \tau+d}\right)=(c \tau+d)^{w} G_{w}(\tau), \quad 4 \leq w \in 2 \mathbb{Z},
$$

and the ring of all holomorphic modular forms is generated by $G_{4}$ and $G_{6}$. The Eisenstein series

$$
E_{2}(\tau)=\frac{3}{\pi^{2}} G_{2}(\tau)=D \varphi(\tau)=\frac{1}{2 \pi i} \frac{d \varphi}{d \tau}
$$

derives from the logarithm of Dedekind's eta function [3],

$$
\begin{aligned}
& \varphi(\tau)=24 \log \eta(\tau)=\log \Delta(\tau), \\
& \eta(\tau)=q^{1 / 24} \prod_{n=1}^{\infty}\left(1-q^{n}\right) .
\end{aligned}
$$

Cusp forms are modular forms that vanish when $\tau \rightarrow i \infty$, and the discriminant

$$
\Delta(\tau)=\left(g_{2}^{3}-27 g_{3}^{2}\right) /(2 \pi)^{12}=\eta^{24}(\tau)
$$

of the elliptic curve is the unique modular cusp form of weight 12 , up to normalization. Since 


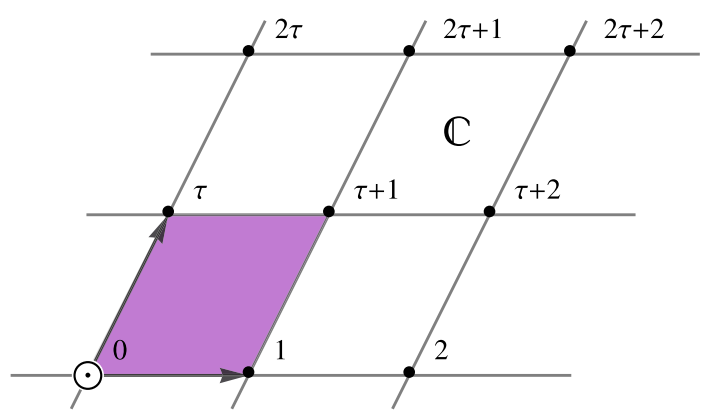

Figure I. The two-dimensional lattice $\Lambda_{\tau} \subset \mathbb{C}$ is generated by the vectors $(1,0)$ and $(0, \tau)$ spanning the fundamental lattice cell (shaded purple). The punctured lattice $\Lambda_{\tau}^{\prime}$ is $\Lambda_{\tau}$ with the origin $\odot$ removed. Each horizontal string of lattice points is a one-dimensional sublattice that we will call a chain, whence $\Lambda_{\tau}$ may be parsed as a stack of chains.

$$
\eta(\tau+1)=e^{i \pi / 12} \eta(\tau), \quad \eta(-1 / \tau)=\sqrt{-i \tau} \eta(\tau),
$$

the Dedekind eta function transforms almost (i.e., up to phases) like a modular form of weight $1 / 2$ on $\Gamma$, and it is a modular form of weight $1 / 2$ on the metaplectic double cover of $\Gamma$. It has many subtle and surprising connections to other areas of mathematics, including number theory, topology, index theory of elliptic operators, algebraic geometry, and gauge theory [2].

\section{Quasimodular Planar Cubics}

Because the sum defining $G_{2}$ in (3) is not uniformly convergent, it does not transform like a modular form as in (4),

$$
G_{2}\left(\frac{a \tau+b}{c \tau+d}\right)=(c \tau+d)^{2} G_{2}(\tau)-2 \pi i c(c \tau+d) .
$$

This had to be the case, since no modular form on $\Gamma$ of weight less than four exists. Rather, $i G_{2} / \pi$ transforms like a connection on the modular curve $\mathcal{X}$, which is $\mathbb{C}^{+} / \Gamma$ compactified by gluing the rational numbers to the upper halfplane, called a quasimodular form of weight two. It is implicit in the work of Weierstrass, as will be explained below, but quasimodular forms were first studied systematically by Ramanujan [9], who called them mock theta functions, and a decade later by Hecke [6].

This lack of modularity is why Weierstrass removes the constant term $G_{2}$ from the Laurent expansion of the wouldbe modular $\wp$ on a disk punctured at the origin, leaving

$$
\wp(z ; \tau)=\frac{1}{z^{2}}+\sum_{k=1}^{\infty}(2 k+1) G_{2 k+2}(\tau) z^{2 k} .
$$

The price he pays for this is that his sigma and zeta functions have parts containing $G_{2}$ that spoil an otherwise perfect analogy with trigonometry (cf. the section on lattice functions below).

Let us consider what happens to the planar Weierstrass cubic in (1) if we put the constant term back in the game,

$$
\wp_{2}(z ; \tau)=\wp(z ; \tau)+G_{2}(\tau) .
$$

Using the Ramanujan identities [9]

$$
\begin{aligned}
12 D E_{2} & =E_{2}^{2}-E_{4}, \\
3 D E_{4} & =E_{2} E_{4}-E_{6}, \\
2 D E_{6} & =E_{2} E_{6}-E_{4}^{2}
\end{aligned}
$$

(a geometric interpretation of these may be found in the appendix), we find that every elliptic curve is isomorphic to a quasimodular planar cubic

$$
\begin{aligned}
\left(d \wp_{2} / d z\right)^{2} & =4 \wp_{2}^{3}-g_{1} \wp_{2}^{2}-\partial g_{1} \wp_{2}-\partial^{2} g_{1} / 6 \\
& =4 \wp_{2}^{3}+\partial \varphi \wp_{2}^{2}+\partial^{2} \varphi \wp_{2}+\partial^{3} \varphi / 6
\end{aligned}
$$

in $\mathbb{C P}_{2}\left[\wp_{2}, d \wp_{2} / d z\right]$. Surprisingly, the coefficients in this curious cubic are simply derivatives $\partial \varphi=2 \pi i d \varphi / d \tau=$ $-g_{1}=-12 G_{2}$, etc., of the logarithm of Dedekind's $\eta$-function.

Thus, every elliptic curve is "contained" in the logarithm of the Dedekind $\eta$-function (or equivalently, the logarithm of the discriminant), since it is completely determined by the first three derivatives of $\log \eta$ (or $\log \Delta$ ) evaluated at the point $\tau$ on the modular curve that gives the shape of the elliptic curve (up to modular transformations).

What follows is a more or less self-contained introduction to elliptic functions, which will enable us to derive (9) directly from first principles, i.e., without the aid of Weierstrass theory. The presentation is not the conventional one found in textbooks, but it is arguably a more intuitive approach that may serve as a precursor to the classical theory. ${ }^{2}$ It is also better adapted to the task of studying pinched tori, i.e., the large complex structure limit $\tau \rightarrow i \infty$, where the tension between holomorphy and automorphy (modular symmetry) that infects modular mathematics becomes acute. This should not be suppressed, as is usually done, but confronted head on from the beginning so that we can see how it fits into the story. Anomalous symmetries play a fundamental role in quantum field theory, and we shall see that quasimodular symmetries and the associated holomorphic anomaly are equally important in understanding properties of singular geometries.

\section{Elliptic Functions}

Periodic (circle) functions are called trigonometric functions. A product of two circles is a torus, which after a point of origin has been chosen is called an elliptic curve. If we do not impose any constraints, it is way too easy to make doubly periodic functions: the product $P(x) Q(y)$ of any two periodic functions $P$ and $Q$ is doubly periodic, frequently

${ }^{2}$ This presentation is, at least in spirit, much closer to Eisenstein's original work than to the subsequent (and now universally adopted) approach taken by Weierstrass $[4,10]$. 
finite, but never (by Liouville's theorem) holomorphic (complex analytic), except for constants; cf. Figure 2(a). The useful compromise is to consider meromorphic doubly periodic (toroidal) functions, which are called elliptic functions.

The minimum amount of divergence is to have a double pole (which may be split into two simple poles) per lattice cell; cf. Figure 1. The prototypical elliptic function is the Weierstrass $\wp$-function defined in (2), which is plotted on a square torus $(\tau=i)$ in Figure 2(b), and on a rectangular torus with $\tau=2 i$ in Figure 2(c). If the real part $\mathfrak{R}_{\wp}$ or imaginary part $\mathfrak{I} \wp$ is plotted instead of (as here) $|\wp|$, then it is obvious that it has double poles; cf. Figures 5 and 6 .

We can regard both periodic and doubly periodic functions as lattice functions, i.e., lattice sums that are manifestly periodic in one or two directions. Furthermore, we shall view a two-dimensional (2D) lattice as a stack of one-dimensional (1D) lattices, each of which is a string of lattice points parallel to the real line (cf. Figures 1 and 2 ) that we call a chain. We can dissect $2 \mathrm{D}$ sums by doing one chain at a time, and we therefore suspect that elliptic and trigonometric functions are close cousins. That this is indeed the case is most easily seen by constructing both as lattice sums. For example, we shall soon see that the closest cousin to $\wp(z)$ is $\pi^{2} \csc ^{2} \pi z$.

A chain is a very specific horizontal linear string of points that stay together when the complex structure $\tau$ of the torus is changed. This changes how far apart the chains are, but they do not change shape, and it is therefore natural to treat them as building blocks of the 2D lattice; cf. Figures 1, 2, and 6 .

This carries over to chain functions, which are 1D lattice sums of rational functions that define trigonometric functions. When these functions are complexified, the chain functions are glued together by the complex structure, but they retain their identity as building blocks of elliptic functions.

This parsing of a 2D lattice as a stack of chains highlights the similarities between 1D and 2D lattice functions, and it is the main pedagogical device used here to explain that elliptic lattice functions (rather than Weierstrass functions) are the closest relatives of trigonometric functions.

Our convention is that the lattice $\Lambda_{\tau}$ is $1 \mathrm{D}$ with period $\tau$ if $\tau$ is a positive real number, i.e., $\Lambda_{\tau}=\tau \mathbb{Z}$ when $\tau \in \mathbb{R}^{+}$. The lattice $\Lambda_{\tau}$ is $2 \mathrm{D}$ with basis $(1, \tau)$ if $\tau$ is not real $(\mathfrak{I} \tau \neq 0)$, and we can without loss of generality parameterize all such lattices by the upper complex half-plane $\mathbb{C}^{+}=\{\tau \in \mathbb{C} \mid \mathfrak{I} \tau>0\}$

$$
\Lambda_{\tau}=\mathbb{Z} \times \tau \mathbb{Z}=\left\{m+n \tau \mid \tau \in \mathbb{C}^{+} ; m, n \in \mathbb{Z}\right\} .
$$

Doubly periodic functions are obtained by tracing fractions (rational functions) over a $2 \mathrm{D}$ lattice $\Lambda_{\tau \notin \mathbb{R}}$.

\section{Chain Functions}

We first consider 1D periodic functions from the lattice point of view, preparing a template onto which we later can graft doubly periodic functions. In other words, we

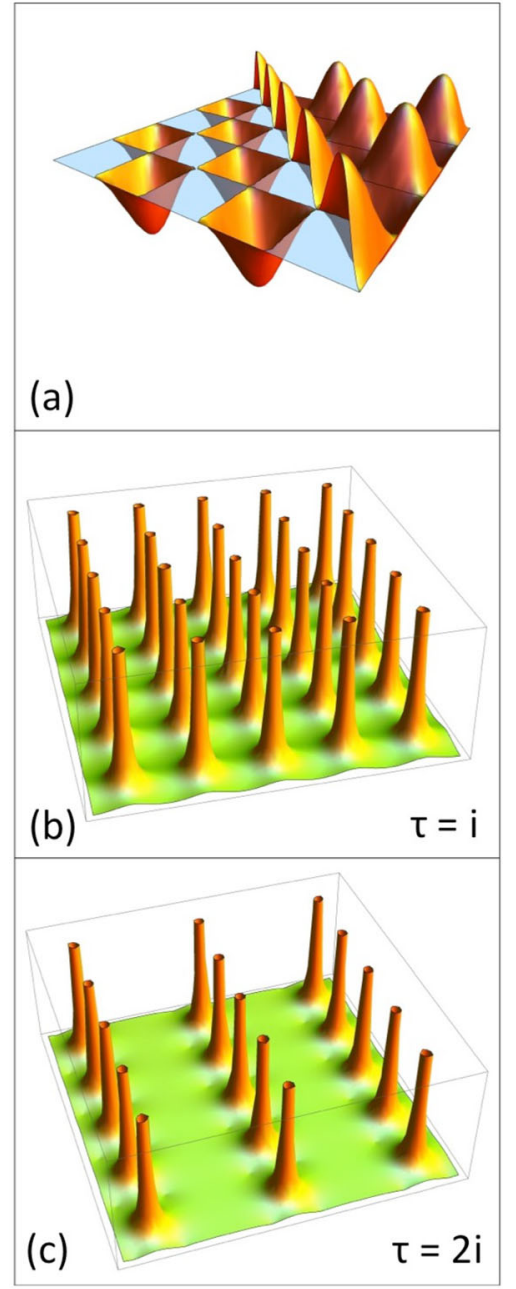

Figure 2. Doubly periodic functions are rarely elliptic. (a) The real doubly periodic function $\sin x \sin y$, with some of the graph removed to reveal the checkerboard symmetry. This is not an elliptic function. The Weierstrass function $\wp(z ; \tau)$ is elliptic, with one double pole on each lattice cell: (b) $|\wp(z ; i)|$ (square torus), (c) $|\wp(z ; 2 i)|$ (rectangular torus). Notice how strings of lattice points move apart as $\mathfrak{I} \tau$ increases, eventually becoming individual 1D lattices that we here call chains.

want to have a unified treatment of multiperiodic functions that exposes cross-dimensional similarities, which can then be exploited in the construction of elliptic functions. Our aim is to be as concrete and explicit as possible, using only elementary analysis and checking results by plotting them. Subtle convergence questions (convergence, absolute convergence, or uniform convergence) are effectively avoided by exploiting well-known analytic properties of special (zeta and polygamma) functions.

The lattice is $\Lambda_{1}=\mathbb{Z}$. The choice of origin on the lattice is arbitrary, but an origin is required in order to have a group structure on the lattice (elliptic curves are abelian groups). Functions that are manifestly periodic can be obtained by cloning a rational function on each link. The simplest seed function that has any chance of giving a convergent sum is $x^{-n}$ for sufficiently large $n$, which is 
copied onto each link of the lattice $\Lambda_{1}=\mathbb{Z}$ by translation. The trace over the lattice is a superposition of these link functions, i.e., the lattice sum

$$
p_{n}(x)=\sum_{m \in \mathbb{Z}} \frac{1}{(x+m)^{n}}, \quad x \in \mathbb{R} \backslash \mathbb{Z},
$$

which has poles of order $n$ when $x \in \mathbb{Z}$. Since the sum is absolutely convergent when $n \geq 2$, it can be differentiated term by term, giving the recurrence relation $(d / d x$ is abbreviated as $\partial_{x}$ )

$$
p_{n+1}=-\frac{1}{n} \partial_{x} p_{n}=\frac{1}{n !}\left(-\partial_{x}\right)^{n-1} p_{2}, \quad n \geq 1 .
$$

So we need to compute only $p_{2}$, but it is instructive to calculate all the $p_{n}$ directly, using a method that is tailormade for the analytic regularization that will follow.

For $n \geq 2$, the Riemann-Hurwitz zeta function is

$$
\zeta(n, x)=\sum_{m=0}^{\infty} \frac{1}{(x+m)^{n}}=\frac{(-1)^{n}}{\Gamma(n)} \psi^{(n-1)}(x),
$$

where $\psi^{(n)}(x)=\partial_{x}^{n+1} \log \Gamma(x)(n \geq 0)$ is the polygamma function. Equation (10) can therefore be written as

$$
\begin{aligned}
p_{n}(x) & =\zeta(n, x)+(-1)^{n} \zeta(n, 1-x) \\
& =\frac{1}{\Gamma(n)}\left[\psi^{(n-1)}(1-x)+(-1)^{n} \psi^{(n-1)}(x)\right] .
\end{aligned}
$$

Using that the polygamma function satisfies the reflection formula

$$
(-1)^{n} \psi^{(n)}(1-x)=\psi^{(n)}(x)+\pi \partial_{x}^{n} \cot \pi x, \quad n \geq 0,
$$

we obtain

$$
p_{n}(x)=\frac{\pi}{\Gamma(n)}\left(-\partial_{x}\right)^{n-1} \cot \pi x, \quad n \geq 2 .
$$

As expected, all these periodic functions are trigonometric.

We would like to extend this result to the case $n=1$, but it is not even obvious that $p_{1}$ is well defined, since the zeta function has its only pole at $n=1$. Whenever we encounter a sum of dubious convergence, we must regulate it. ${ }^{3}$ Regularization is a procedure that extracts a finite part from a (potentially) divergent series. As long as the procedure satisfies the Hardy axioms [5], the result should be independent of the chosen regularization scheme. The most popular schemes (in both physics and mathematics) are cutoff regularization and analytic regularization.

A cutoff scheme makes everything explicitly finite at every step until the limit is taken at the end. However, a cutoff is a discrete regulator that typically breaks every symmetry in sight. For example, the lattice $\Lambda_{1}=\mathbb{Z}$ is invariant under translations $T(x)=x+1$, since we can relabel all lattice points, but note that this works only because the lattice is infinite in both directions. No points "drop off the end" when shifted left or right, because there are no end points. If the lattice sum is regularized by chopping off all but a finite piece in the middle, as is often done, then this symmetry is brutally violated. Since our motivation for tracing over infinite lattices is to build manifestly periodic functions, it is not very appealing to immediately destroy this circle symmetry by cutting off the sum at both ends in order to make it finite, even if periodicity is eventually restored. We prefer a more benign scheme that respects symmetries.

The finiteness of analytic schemes is not as explicit, but they are continuous and do minimal harm to the geometric structure of the theory. In particular, they respect all symmetries as far as possible. ${ }^{4}$ Since symmetry is the DNA of any quantum theory, analytic schemes are usually preferred in physics. They are essentially variations on Riemann's analytic continuation of the series that defines his zeta function: embed the sum into a larger analytic family, using a reflection formula if possible, and then define the value of the suspicious series to be the limit of a sequence of wellbehaved family members. Here we need only the (generalized) Riemann zeta function.

Sometimes it is not possible to regulate a function (theory) without harming its geometric structure, i.e., without breaking some symmetry. This generates so-called anomalies that carry subtle and useful information about the geometric structure. In the theory of elliptic functions, they give an explicit parameterization of the conflict between holomorphy and automorphy suffered by modular forms of low weight. If it is impossible to maintain both a holomorphic structure and modular symmetry, then the modular anomaly explicitly exhibits the problem, and it can be used to construct functions that respect one or the other of these geometric structures, but not both simultaneously.

This will play an important part in our discussion, since we are particularly interested in the boundary of moduli space, where this problem becomes acute. ${ }^{5}$ In order to analyze the so-called nodal limit, in which a torus is pinched, we need a holomorphic function that transforms anomalously under modular transformations. It is nearly a weight-two form, and it emerges naturally from lattice sums that are convergent, but not uniformly convergent. This subtle aspect of infinite series is respected by our analytic regulator, and the required anomaly appears automatically. This is a good reason to use an analytic regulator. If a cutoff scheme produces an anomaly, how can we be absolutely sure that this is not an artifact of the explicit symmetrybreaking introduced by the regularization scheme? How do

\footnotetext{
${ }^{3}$ Regularizing a quantum field theory, which usually is rife with infinities, is a prerequisite for the infamous procedure called renormalization. This extracts the physical content from apparently ill-defined expressions and may be regarded as a very adult version of our discussion of $p_{1}$.

${ }^{4}$ Classical symmetries are sometimes broken by quantum fluctuations. If this anomalous symmetry is local (gauge invariance), then this is fatal, and the quantum field theory is discarded, but anomalous global symmetries may be tolerable (since global symmetries typically are broken in the real world), and even useful.

${ }^{5}$ The original physical motivation is that this is the weak coupling limit of a particular effective quantum field theory that captures universal properties of quantum Hall systems. This theory is a nonlinear sigma model with a toroidal target space, which degenerates to a nodal curve in the perturbative domain [7, 8]. Conventional quantum perturbation theory is a nonmodular asymptotic expansion adapted to the singular geometry. The broken (or hidden) modular symmetry is presumably related to the holomorphic anomaly.
} 
we know that other symmetry-violating schemes do not produce different anomalies? The anomaly would then not be canonical, and would not carry any invariant geometric or topological information.

So, since the zeta function can be analytically continued to $\mathbb{C} \ni s \neq 1$, we investigate the analytic continuation

$$
p_{s}(z)=\sum_{m \in \mathbb{Z}} \frac{1}{(z+m)^{s}}=\zeta(s, z)+(-1)^{s} \zeta(s, 1-z)
$$

of $p_{n}$ in the limit as $s \rightarrow 1$. If $p_{1}$ is well defined, the poles of the two zeta functions must cancel, and they do:

$$
\begin{aligned}
p_{1}(x) & =\lim _{s \rightarrow 1} p_{s}(x) \\
& =\lim _{s \rightarrow 1}\left[\frac{1}{s-1}-\psi^{(s-1)}(x)-\frac{1}{s-1}+\psi^{(s-1)}(1-x)\right] \\
& =\psi(1-x)-\psi(x)=\pi \cot \pi x,
\end{aligned}
$$

where use has been made of the reflection property of the digamma function $\psi(x)=\psi^{(0)}(x)$ recorded in (11).

Equation (12) can therefore be extended to include the case $n=1$, since this also gives $p_{1}(x)=\pi \cot \pi x$. Finally, since $p_{1}(x)=-\partial_{x} p_{0}(x)$ with $p_{0}(x)=-\log \sin \pi x$, we can obtain all $p_{n}$ from the "potential" $p_{0}$ by derivation:

$$
p_{n}(x)=\frac{(-1)^{n}}{\Gamma(n)} \partial_{x}^{n} p_{0}(x), \quad n \geq 1 .
$$

As a final check, we expand the $p_{n}(x)$ defined by (10) for $|x|<1$

$$
\begin{aligned}
p_{n}(x) & =\frac{1}{x^{n}}+\sum_{k=0}^{\infty} \gamma_{n+k}\left(\begin{array}{c}
n+k-1 \\
n-1
\end{array}\right)(-x)^{k}, \\
\gamma_{n+k} & =\sum_{m \in \Lambda_{1}^{\prime}} \frac{1}{m^{n+k}}, \quad \Lambda_{1}^{\prime}=\mathbb{Z} \backslash\{0\} .
\end{aligned}
$$

We observe that odd coefficients $(n+k=2 r+1)$ vanish, while even coefficients $(n+k=2 r)$ are zeta functions,

$$
\gamma_{2 r}=\sum_{m \in \Lambda_{1}^{\prime}} \frac{1}{m^{2 r}}=2 \zeta(2 r) .
$$

Using that $\zeta(0)=-1 / 2$, we find that $p_{1}(x)$ must be the cotangent function,

$$
p_{1}(x)=-\sum_{r=0}^{\infty} \gamma_{2 r} x^{2 r-1}=-2 \sum_{r=0}^{\infty} \zeta(2 r) x^{2 r-1}=\pi \cot \pi x v,
$$

since it is well known that this is a generating function for zeta functions. Notice also that

$p_{2}(x)=\frac{1}{x^{2}}+\sum_{k=0}^{\infty}(2 k+1) \gamma_{2 k+2} x^{2 k}=\frac{1}{x^{2}}+\gamma_{2}+3 \gamma_{4} x^{2}+\cdots$.

Comparison with (6) and (7) shows that the 1D lattice functions $\gamma_{w}=2 \zeta(w)$ on $\Lambda_{1}^{\prime}$ are analogous to the 2D lattice functions $G_{w}(\tau)$ on $\Lambda_{\tau}^{\prime}$ in Weierstrass theory. Not surprisingly, from (3) we see that $\gamma_{w}$ is in fact the leading $(\tau$ independent) term in the $q$-expansion of $G_{w}$, so we are on the right track. Since $p_{n}(x)=(-1)^{n} p_{n}(-x)$, these periodic lattice functions are parity eigenstates, and we have found the two complete families of odd/even functions shown in Figure 3.

Equation (13) gives a hierarchy of algebraic and differential equations:

$$
\begin{aligned}
& p_{0}=-\log \sin \pi x, \\
& p_{1}=-p_{0}^{\prime}=\pi \cot \pi x, \\
& p_{2}=-p_{1}^{\prime}=p_{1}^{2}+\pi^{2}=\pi^{2} \csc ^{2} \pi x, \\
& p_{3}=-\frac{1}{2} p_{2}^{\prime}=\frac{1}{2} p_{1}^{\prime \prime}=p_{1} p_{2}=\pi^{3} \cot \pi x \csc ^{2} \pi x, \\
& p_{4}=-\frac{1}{3} p_{3}^{\prime}=\frac{1}{6} p_{2}^{\prime \prime}=p_{2}\left(p_{2}-\frac{2 \pi^{2}}{3}\right), \\
& p_{5}=-\frac{1}{4} p_{4}^{\prime}=p_{1} p_{2}\left(p_{2}-\frac{\pi^{2}}{3}\right),
\end{aligned}
$$

where $p_{0}^{\prime}=\partial_{x} p_{0}=d p_{0} / d x$, etc. The two expressions for $p_{4}$ give a second-order differential equation for $p_{2}$, while a first-order equation is obtained by squaring $p_{3}$ and using that $p_{1}^{2}=p_{2}-\pi^{2}$,

$$
p_{3}^{2}=\frac{1}{4} p_{2}^{\prime}(x)^{2}=p_{1}^{2} p_{2}^{2}=p_{2}^{2}\left(p_{2}-\pi^{2}\right) .
$$

This is a cubic equation in (renormalized) chain functions, which with $(X, Y)=\left(p_{2} / \pi^{2}, p_{3} / \pi^{3}\right)$ is the cubic ${ }^{6}$

$$
Y^{2}=X^{2}(X-1)=\left(X^{\prime}\right)^{2} / 4 \subset \mathbb{R}^{2} .
$$

Although this looks like a planar cubic (a nodal curve in the complex projective plane $\mathbb{C P}_{2}$ ), as a real equation it is equivalent to the conventional quadratic equation $u^{2}+$ $v^{2}=1$ that embeds the circle in the real plane $\mathbb{R}^{2}(u, v)$. At the moment, this just looks like a very clumsy way of writing trigonometric identities, but when these functions are promoted to elliptic functions, they will become nontrivial statements. The corresponding elliptic equation embeds a pinched torus in the complex projective plane $\mathbb{C P}_{2}$.

\section{Stereography of Circles}

We give a geometric interpretation of real chain functions and the algebraic equations they satisfy that will be useful later. Consider first the projective representation of the circle $Y^{2}=X(1-X) \subset \mathbb{R}^{2}$ shown in Figure 4. Every point $(X, Y)$ on this circle, except the origin, is projected onto the line $X=1$ by finding the point $(1, c)$ on the line where a ray from the origin through $(X, Y)$ intersects it. ${ }^{7}$ If the ray makes an angle $\varphi$ with the $Y$-axis, then $c=\cot \varphi$, and the

${ }^{6}$ Notice that the "Weierstrassian" choice $Y_{W}=p_{2}^{\prime} / \pi^{3}=-2 Y$ would give $Y_{W}^{2}=4 X^{2}(X-1)$. The analogous construction in the elliptic case explains the factor of 4 in (1).

${ }^{7}$ In Figure 4, this line is called a screen, by which we mean a one- or two-dimensional manifold onto which another shape is projected. In two dimensions, discussed in the section on pinched nodal cubics below, the analogous "projection screen" is a 2D plane, for which the terminology is more appropriate. 

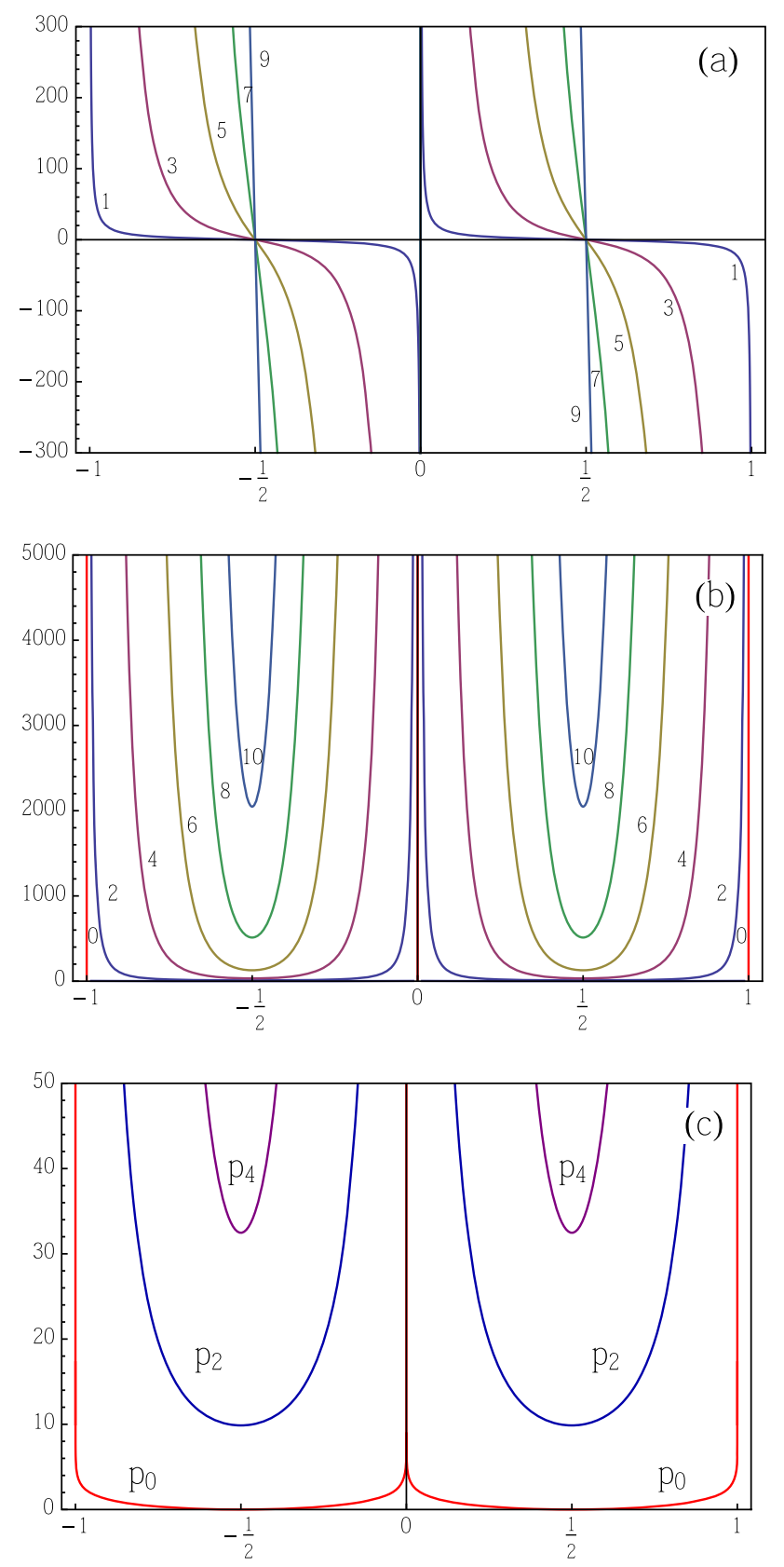

Figure 3. The periodic lattice functions split into two families. (a) $p_{n}$ is odd for odd $n \geq 1 ; p_{1}$ fits snugly into this family, as it should if the lattice trace has been properly regularized. (b) $p_{n}$ is even for even $n \geq 0$. (c) Magnification of (b) showing how the potential $p_{0}$ fits into the even family.

projection is given by

$$
(X, Y) \stackrel{\Pi_{1}}{\longmapsto}(1, c)=(1, Y / X)=(1, \cot \varphi) .
$$

The inverse projection is obtained by inserting $Y=c X$ into the quadric $Y^{2}=X(1-X)$,

$$
(1, c) \stackrel{\Pi_{1}^{-1}}{\longmapsto}(X, Y)=\frac{1}{1+c^{2}}(1, c)=\left(\sin ^{2} \varphi, \frac{\sin 2 \varphi}{2}\right) .
$$

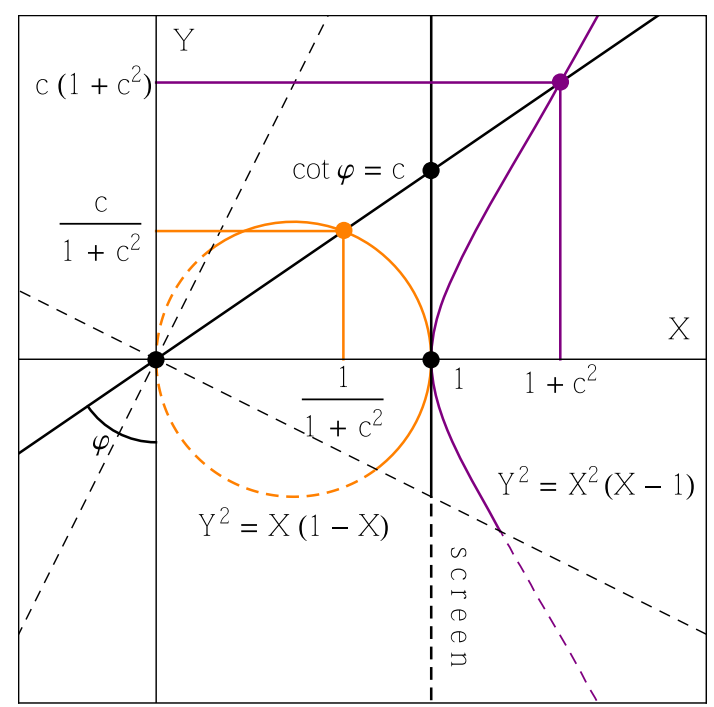

Figure 4. Stereography of real quadrics and cubics. Simultaneous parametric plot of the quadric $Y^{2}=X(1-X)$ (orange) and the cubic $Y^{2}=X^{2}(X-1)$ (purple). Solid curves have $c \in[-1 / 2,2]$, while the dashed parts of the curves are obtained for other values of $c$, i.e., $c \in\langle-\infty,-1 / 2\rangle \cup\langle 2, \infty\rangle$. A pair of points on the quadric (orange) and cubic (purple) parameterized by $c$ both project to the same point $(1, c)$ (black) on the line $x=1$, here called a (projection) screen. The point $(0,0)$ on the circle projects to $i \infty$ on the screen and on the cubic, which are compactified by adding this point.

Note that both the projection and its inverse are rational transformations. The line $X=1$ is therefore birational to the circle almost everywhere. The exception is the point $(X, Y)=(0,0)$, where the transformations are singular. In order that this point also have an image on the real line, a single new point at infinity is added to the screen $X=1$, which is thereby closed at infinity to form a topological circle. In short, the full circle is birational to the compactified line, and no structure has been lost by this procedure.

Consider next the real cubic given by (14), which is also plotted in Figure 4. Since it is built from periodic (circle) functions, we expect it to be related to a circle. It does not look much like a circle, but neither did the line $X=1$ in the example above. In order to see what is going on, we also project the cubic onto this line. Using again $y=c x$, we get

$$
\begin{aligned}
& (X, Y) \stackrel{\Pi_{2}}{\longmapsto}(1, c)=(1, Y / X), \\
& (1, c) \stackrel{\Pi_{2}^{-1}}{\longmapsto}(X, Y)=\left(1+c^{2}\right)(1, c)=\left(\csc ^{2} \varphi, \cot \varphi \csc ^{2} \varphi\right) .
\end{aligned}
$$

With the identification $\varphi \leftrightarrow \pi x$, we see that we have recovered the functions $p_{2}=\pi^{2} X=\pi^{2} \csc ^{2} \varphi$ and $p_{3}=$ $\pi^{3} Y=\pi^{3} \cot \varphi \csc ^{2} \varphi$ previously obtained from lattice sums. In other words, the lattice functions are a kind of stereographic coordinates for this real cubic curve. A similar result in the complex case will illuminate the nodal limit. 
Since both $\Pi_{1}$ and $\Pi_{2}$ are birational transformations, so are compositions of these maps:

$$
\Pi_{2}^{-1} \circ \Pi_{1}(X, Y)=\frac{X^{2}+Y^{2}}{X^{3}}(X, Y)
$$

is a rational map of the quadric to the cubic, and

$$
\Pi_{1}^{-1} \circ \Pi_{2}(X, Y)=\frac{X}{X^{2}+Y^{2}}(X, Y)
$$

is a rational map of the cubic to the quadric. This shows that this cubic is a birational projective representation of the circle.

In short, by gluing in the point at infinity, the cubic is compactified and given the topology of a circle. When we consider tori, this will become more explicit. Taking the two simplest slices of the torus gives two orthogonal circles that can be used to generate the torus, and in the projective picture, one of them typically looks like the cubic curve in Figure 4.

The manner in which a real cubic can faithfully represent a circle is closely related to how a pinched torus can represent a sphere. The analogous result in $2 \mathrm{D}$ is that a planar nodal cubic (a degenerate complex cubic equation in the complex projective plane $\mathbb{C P}_{2}$ ) is stereographically birational to the complex line $\mathbb{C P}_{1} \simeq S^{2}$, i.e., a sphere.

In summary, chain sums give a simple family of periodic functions that may be derived from the potential function $p_{0}(x)=-\log \sin \pi x$ (by derivation), which is perfectly adapted to (real) stereography. The main virtue of this approach to trigonometry is that it immediately generalizes to stacks of chains, i.e., 2D lattices, i.e., elliptic functions. In doing so, the large complex structure limit of the torus, where it is pinched down to a topological sphere, is illuminated, thereby clarifying the somewhat mysterious modular/holomorphic anomaly that gives rise to quasimodular (mock modular) forms that have recently resurfaced in various contexts.

\section{Lattice Functions}

Elliptic functions can be constructed from the simplest lattice sums in 2D, regularized using 2D "zeta functions," in close analogy with the 1D case. Note, however, that while a $2 \mathrm{D}$ lattice is exactly the same as a stack of 1D lattices or chains (cf. Figure 1), elliptic (2D) lattice functions are not simply stacks of real chain functions (cf. the section on elliptic functions above). When chain functions are complexified, they become glued together by the complex structure of the 2D manifold to form meromorphic functions. The sums

$$
\wp_{n}(z ; \tau)=\sum_{w \in \Lambda_{\tau}} \frac{1}{(z+w)^{n}}, \quad n \geq 3,
$$

where $\Lambda_{\tau}$ is the lattice shown in Figure 1, are explicitly doubly periodic and meromorphic (elliptic) when they are well defined, i.e., absolutely convergent $(n \geq 3)$. We wish to expand this family of functions to the missing cases

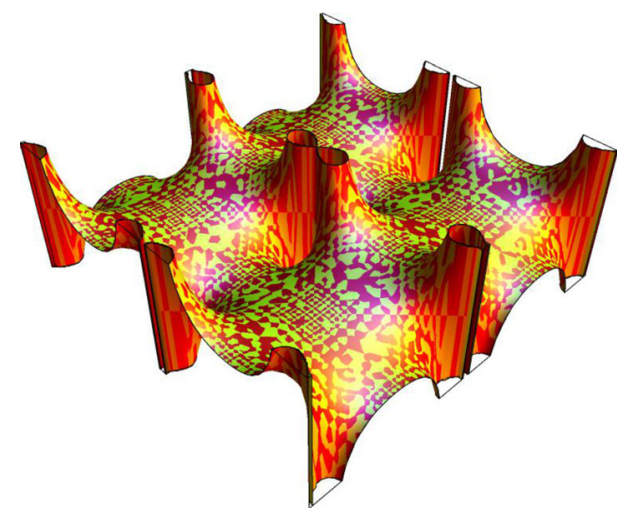

Figure 5. The Weierstrass function $\mathfrak{R}_{\wp}(z ; \tau)$ (green) plotted on top of the stack $\mathfrak{R}\left[\wp_{2}(z, \tau)-G_{2}(\tau)\right]$ (red) of complex trigonometric chains $p_{2}(z+m \tau)=\pi^{2} \csc ^{2} \pi(z+m \tau)$, with $m=-2$, $-1,0,1,2$ and $\tau=i$. The quilt appears because of tiny random numerical differences between the two functions. Compare also Figure 6, where the chain stacking is investigated in more detail.

$n=0,1,2$, and more generally to a meromorphic function $\wp_{s}(z ; \tau)$, using analytic continuation.

The recurrence relation is as before,

$$
\wp_{n+1}(z ; \tau)=-\frac{1}{n} \frac{d}{d z} \wp_{n}(z ; \tau)=\frac{1}{n !}\left(-\frac{d}{d z}\right)^{n} \wp_{1}(z ; \tau),
$$

where $\wp_{1}$ is given by a $q$-expansion,

$$
\begin{aligned}
\wp_{1}(z ; \tau) & =p_{1}(z)+S_{1}(z ; \tau), \\
S_{1}(z ; \tau) & =\sum_{m=1}^{\infty}\left[p_{1}(z-m \tau)+p_{1}(z+m \tau)\right] \\
& =2 \pi i \sum_{m=1}^{\infty}\left[\frac{1}{q^{m} u-1}-\frac{1}{q^{m} / u-1}\right],
\end{aligned}
$$

with $q=\exp (2 \pi i \tau)$ and $u(z)=\exp (2 \pi i z)$. In the last line, we used that $p_{1}(z)=\pi \cot \pi z$, derived with great care above in the section on chain functions. Feeding this back into the recurrence relation gives

$$
\wp_{n+1}(z ; \tau)=p_{n+1}(z)+\frac{1}{n !}\left(-2 \pi i u \frac{d}{d u}\right)^{n} S_{1}(z ; \tau) .
$$

For $|z|<1$ we can expand $\wp_{n}$ in a power series:

$$
\begin{aligned}
\wp_{n}(z ; \tau) & =\frac{1}{z^{n}}+\sum_{m=0}^{\infty} G_{n+m}(\tau)\left(\begin{array}{c}
n-1+m \\
n-1
\end{array}\right)(-z)^{m}, \\
G_{n}(\tau) & =\sum_{w \in \Lambda_{\tau}^{\prime}} \frac{1}{w}=\sum_{m \in \Lambda_{1}^{\prime}} \sum_{k \in \Lambda_{1}} \frac{1}{(k+m \tau)^{n}},
\end{aligned}
$$

where the $G_{n}$ by definition are the expansion coefficients of $\wp_{1}$. Since $G_{n}=(-1)^{n} G_{n}$, these coefficients vanish for odd $n$. For even $n$, we evaluate them using $\zeta$-functions [1],

$$
G_{n}(\tau)=2 \zeta(n)+2 \frac{(2 \pi i)^{n}}{\Gamma(n)} \sum_{k=1}^{\infty} \frac{k^{n-1} q^{k}}{1-q^{k}} .
$$

The real part of the Weierstrass function is very similar to the $1 \mathrm{D}$ lattice function $p_{2}(x)-\pi$. They are by construction 
close relatives, but they are not the same, because the other chains contribute a small amount also for real arguments.

Consider,

for

example,

$p_{2}(1 / 2)-\pi=\pi(\pi-1)=6.72801$, which should be compared to

$$
\begin{aligned}
\wp(1 / 2 ; i) & =\wp_{2}(1 / 2 ; i)-G_{2}(i) \\
& =\pi^{2} \sum_{m \in \mathbb{Z}} \operatorname{sech}^{2} \pi m-\pi=\pi_{\infty}^{2}=6.87519 \ldots,
\end{aligned}
$$

where

$$
\pi_{\infty}=\pi G=\pi \frac{\Gamma(1 / 4)^{2}}{(2 \pi)^{3 / 2}}=2.6220575542 \ldots \approx 21 / 8
$$

is the lemniscate constant, ${ }^{8} G$ is Gauss's constant, and $G_{2}(i)=\pi$ has been used. In order to banish any lingering doubt about the veracity of the stack picture of elliptic functions, both $\wp(z, i)$ and $\wp_{2}(z, i)-\pi$ have been plotted on top of each other in Figure 5. Including only the four chains $m=-2,-1,1,2$ nearest to the $m=0$ chain is sufficient to make the two functions numerically indistinguishable.

We can bring the chain potential $p_{0}(x)=-\log \sin (\pi x)$ into a form suitable for generalization to two dimensions by exponentiating it and using Euler's product formula for the sine function. This leads us to define $\sigma_{\Lambda}$ and $\wp_{0}$ by

$$
\sigma_{\Lambda}(z ; \tau)=e^{-\wp_{0}(z ; \tau)}=\pi z \prod_{w \in \Lambda_{\tau}^{\prime}}\left(1-\frac{z^{2}}{w^{2}}\right) \rightarrow \sin \pi z,
$$

where $z \in \mathbb{C}, w$ take values in a $2 \mathrm{D}$ punctured lattice $\Lambda_{\tau}^{\prime}$ (cf. Figure 1), and the chain limit $\tau \rightarrow i \infty$ is shown. For comparison with Weierstrass, we also relabel $\wp_{1}$ as $\zeta_{\Lambda}$, and $\wp_{2}$ as $\wp_{\Lambda}$ :

$$
\begin{aligned}
& \zeta_{\Lambda}(z ; \tau)=\frac{d}{d z} \log \sigma_{\Lambda}(z ; \tau)=\wp_{1}(z ; \tau) \rightarrow \pi \cot \pi z, \\
& \wp_{\Lambda}(z ; \tau)=-\frac{d}{d z} \zeta_{\Lambda}(z ; \tau)=\wp_{2}(z ; \tau) \rightarrow \pi^{2} \csc ^{2} \pi z .
\end{aligned}
$$

These should be compared with the corresponding Weierstrass functions:

$$
\begin{aligned}
\sigma_{W}(z ; \tau) & =e^{z^{2} \eta_{2}(\tau) / 2-\wp_{0}(z ; \tau)} \rightarrow e^{\pi^{2} z^{2} / 6} \sin \pi z, \\
\zeta_{W}(z ; \tau) & =\frac{d}{d z} \log \sigma_{W}(z ; \tau) \\
& =\wp_{1}(z ; \tau)+z \eta_{2}(\tau) \rightarrow \pi \cot \pi z+\pi^{2} z / 3, \\
\wp(z ; \tau) & =-\frac{d}{d z} \zeta_{W}(z ; \tau) \\
& =\wp_{2}(z ; \tau)-\eta_{2}(\tau) \rightarrow \pi^{2} \csc ^{2} \pi z-\pi^{2} / 3,
\end{aligned}
$$

where $\eta_{2}$ is Weierstrass's eta function, not to be confused with Dedekind's eta function. Comparison with (7) shows that $\eta_{2}(\tau)=G_{2}(\tau)$, which may also be verified by direct calculation.
Figure 6 shows why the Weierstrass function $\wp(z ; \tau)$ may be regarded as a stack $\wp_{2}(z ; \tau)$ of trigonometric chain functions $p_{2}(z+m \tau)$ (compare also Figure 5 ). We see how the Weierstrass function $\wp$ reduces to a single trigonometric chain function $\pi^{2} \csc ^{2} \pi z$ shifted by the anomaly $\pi^{2} / 3$ in the large complex structure limit $\tau \rightarrow i \infty$, where the "bagel" is pinched off to a "bun" (topologically). In other words, the planar cubic degenerates to a nodal curve that is birational to a 2-sphere: $\mathbb{C P}_{2}[3] \longrightarrow \mathbb{C P}_{1} \simeq S^{2}$, as will be discussed in more detail in the section on pinched nodal cubics.

In short, it is the $2 \mathrm{D}$ lattice functions $\wp_{n}$ that enjoy a flawless analogy to trigonometry. The lattice function $\wp_{2}=$ $\wp(z ; \tau)+G_{2}(\tau)$ differs from the Weierstrass function $\wp$ by the modular anomaly $G_{2}$, and this accounts for all the awkward bits in the contrived analogy between Weierstrass theory and trigonometry. This vindicates our starting point, which was to fully exploit the obvious similarities between lattice functions in one and two dimensions (periodic and doubly periodic functions).

Finally, we derive a differential equation satisfied by the lattice functions, using the series expansion in (16). Since $\wp_{2}$ has double poles, the leading-order pole of $\left(d \wp_{2} / d z\right)^{2}$ is $4 / z^{6}$. This can be eliminated by subtracting $4 \wp_{2}^{3}$, leaving a pole of order four, which is eliminated by adding $12 G_{2} \wp_{2}^{2}$, which leaves a pole of order two, which is canceled by subtracting $12\left(G_{2}^{2}-5 G_{4}\right) \wp_{2}$. The series expansion of this pole-free function is

$$
\begin{aligned}
& \left(d \wp_{2} / d z\right)^{2}-4 \wp_{2}^{3}+12 G_{2} \wp_{2}^{2}-12\left(G_{2}^{2}-5 G_{4}\right) \wp_{2} \\
& =4\left(15 G_{2}^{2} G_{4}-G_{2}^{3}-35 G_{6}\right)+\mathcal{O}\left(z^{2}\right),
\end{aligned}
$$

where the remainder $\mathcal{O}\left(z^{2}\right)$ is a regular function. Since the only holomorphic function on a torus is constant (by Liouville's theorem), we have $\mathcal{O}\left(z^{2}\right)=0$. This may also be verified order by order in the series expansion by explicit calculation. Finally, using the Ramanujan identities from (8), derived from the geometry of the modular curve $\mathcal{X}$ in the appendix, we obtain (9). For reasons that soon will be obvious, we shall sometimes refer to this as the stereographic cubic. The Weierstrass cubic in (1) is recovered by substituting $\wp_{2}=\wp+G_{2}$ in (17).

\section{Papillon Plots}

We wish to compare the Weierstrass cubic to the "stereographic" equation solved by the lattice functions $\wp_{2}$ and $\wp_{2}^{\prime}=d \wp_{2} / d z=-2 \wp_{3}$.

While the complexification and projectivization of geometry is the key to modern mathematics, it is not easy to visualize. Even the simple cubic "curve" in the projective "plane" $\mathbb{C P}_{2}$ is really (using real numbers) the intersection of two $3 \mathrm{D}$ hypersurfaces in a $4 \mathrm{D}$ projective space. We therefore introduce a graphical device that goes some way

\footnotetext{
${ }^{8}$ The lemniscate constant $\pi_{\infty}$ (in our nonstandard notation) has gone out of fashion, but it is a fundamental transcendental constant that plays the same role for doubly periodic functions as $\pi$ does for periodic functions. The lemniscate in question appears if you slice a bagel with the knife tangential to the hole. Notice that the rational approximation $\pi_{\infty} \approx 21 / 8$ is surprisingly similar to Archimedes's famous result $\pi \approx 22 / 7$ (sometimes called the engineering value, because it is sufficiently accurate for many practical applications), both good to about one part per thousand.
} 


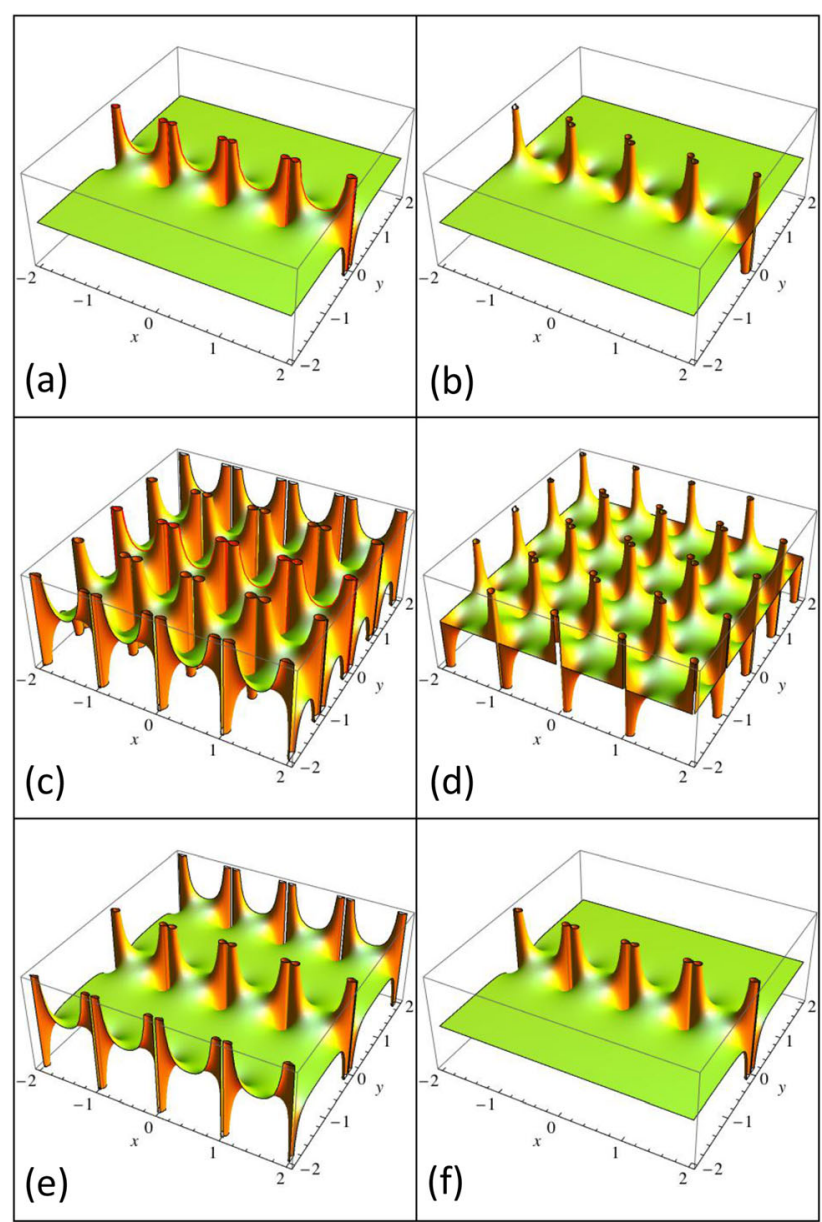

Figure 6. The Weierstrass function $\wp(z ; \tau)$ is the stack $\wp_{2}(z, \tau)$ of complex trigonometric chain functions $p_{2}(z+m \tau)=$ $\pi^{2} \csc ^{2} \pi(z+m \tau) \quad(m \in \mathbb{Z})$, except for the modular anomaly $G_{2}(\tau)$, which has been removed from $\wp$ because it is only quasimodular (compare also Figure 5). (a) The $m=0$ chain shifted by $G_{2}(i)=\pi$ : $\mathfrak{R} p_{2}(z)-\pi=\pi^{2} \mathfrak{R} \csc ^{2} \pi z-\pi$. (b) $\mathfrak{I} p_{2}(z)=\pi^{2} \mathfrak{I} \csc ^{2} \pi z$. (c) $\mathfrak{R} \wp(z ; i)$. (d) $\mathfrak{I} \wp(z ; i)$. (e) $\mathfrak{R}_{\wp}(z ; 2 i)$. (f) $\mathfrak{R}_{\wp}(z ; 4 i) \approx \mathfrak{R} p_{2}(z)-\pi$, since $G_{2}(\tau \rightarrow i \infty) \rightarrow$ $\pi^{2} / 3 \approx \pi$ [compare (a)].

toward visualizing these objects. Clearly, there is no way to retain all information about the cubic in a plot, but a judicious choice of "sections" or "slices" allows us to image the skeleton, i.e., the generating cycles. We consider "family plots" that we shall call papillon plots. The main idea is to "flatten" each pair of cycles onto a plane, and then stack these planes together as one of the parameters of the cubic is changing. They are constructed as follows for the general pure (no mixed terms) cubic

$$
Y^{2}=C(X)=a_{0}+a_{1} X+a_{2} X^{2}+a_{3} X^{3}, \quad X, Y \in \mathbb{C} .
$$

If the coefficients $a_{n}$ are complex, then even this simple curve is too complicated, so we restrict attention to $a_{n} \in \mathbb{R}$. With $X=x_{1}+i x_{2}$ and $Y=y_{1}+i y_{2}\left(x_{1}, x_{2}, y_{1}, y_{2} \in \mathbb{R}\right)$, this is equivalent to the two real equations

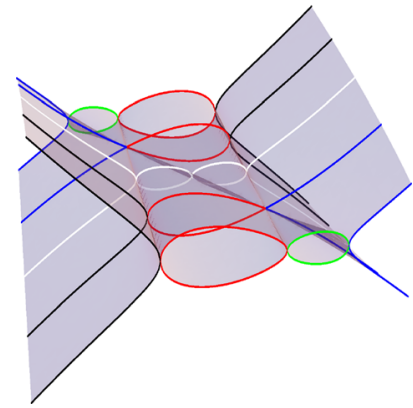

Figure 7. Portrait (papillon plot) of the simple cubic family $y^{2}=x^{3}-a x^{2}+(a-1) x$, with increasing real values of $a$ out of the plane of the paper.

$$
\begin{aligned}
y_{1}^{2}-y_{2}^{2} & =C\left(x_{1}\right)-\left(a_{2}+3 a_{3} x_{1}\right) x_{2}^{2}, \\
2 y_{1} y_{2} & =\left[a_{1}+2 a_{2} x_{1}+a_{3}\left(3 x_{1}^{2}-x_{2}^{2}\right)\right] x_{2} .
\end{aligned}
$$

We obtain maximal simplification by choosing the first section to be $x_{2}=0$. Then either $y_{1}=0$ or $y_{2}=0$, giving two orthogonal sections:

$$
\begin{aligned}
& x_{2}=0=y_{2} \Rightarrow y_{1}^{2}=C\left(x_{1}\right), \\
& x_{2}=0=y_{1} \Rightarrow y_{2}^{2}=-C\left(x_{1}\right) .
\end{aligned}
$$

The 2D papillon (plot of one family member) is obtained by flattening these orthogonal sections into one $2 \mathrm{D}$ diagram composed of four branches (parametric plots):

$$
\mathcal{P}_{2}(x)=\left\{[x, \pm \sqrt{ \pm C(x)}] \mid x \in\left(x_{-}, x_{+}\right)\right\} .
$$

This rotation does not do justice to the geometry of these surfaces, but it does preserve the homotopy, i.e., it respects the topology of the 1-cycles found by sectioning the cubic. A 3D papillon, or family plot, is a two-parameter plot

$$
\mathcal{P}_{3}(x, t)=\left\{[t, x, \pm \sqrt{ \pm C(x, t)}] \mid x \in\left(x_{-}, x_{+}\right), t \in\left(t_{-}, t_{+}\right)\right\} .
$$

The real parameter $t$ is often chosen to be one of the coefficients $a_{i}$, and the other coefficients may also depend parametrically on $t$, i.e., $a_{n}=a_{n}(t)$. As an example of this graphical device, a particularly simple family of cubics is exhibited as a papillon plot in Figure 7.

The top row of Figure 8 shows how the 2D papillon plot develops as the torus is pinched by shrinking a short cycle. The pictures in the middle row are cartoons obtained by "closing the circles at infinity" (where they all meet). The resulting plot is a flattened picture of the cycles exposed by cutting the shapes in the bottom row. Similarly, Figure 9 shows how the 2D papillon plot develops as the torus is pinched by shrinking a long cycle.

\section{Pinched (Nodal) Cubics}

One virtue of the lattice basis is that it allows us to examine in a very explicit way how the torus degenerates to a nodal cubic, which by a stereographic transformation is birationally equivalent to a sphere. 


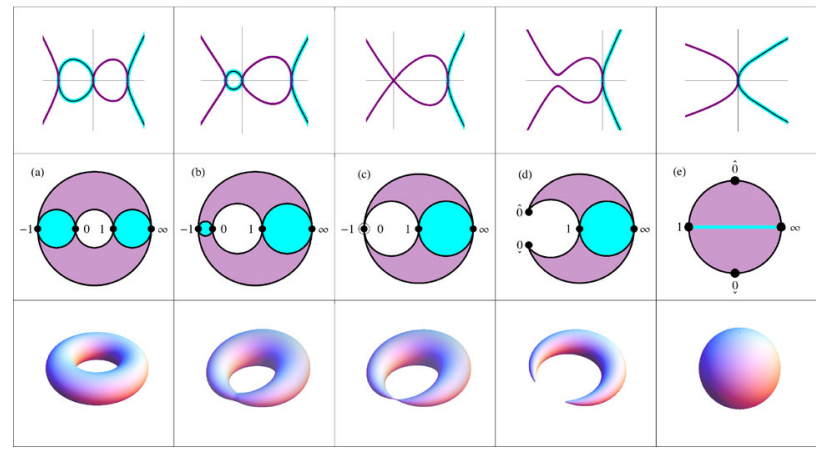

Figure 8. Nodal cubic: shrinking the short cycle.

Consider first the family of rectangular tori parameterized by $\tau=i t$, where $t$ is a positive real number. Since $q(i t)=\exp (-2 \pi t)$ is real, so are all the coefficients of the stereographic cubic in (9) [cf. (3)], and we can construct the papillon plot shown in Figure 10. This plot shows very clearly how a node emerges as $t$ grows larger than 1 and completely dominates the structure of the cubic for $t \gg 1$.

In the (large complex structure) limit $t \rightarrow \infty$, the stereographic cubic reduces to $\wp_{3}^{2}=\wp_{2}^{2}\left(\wp_{2}-\pi^{2}\right)=\left(\wp_{2}^{\prime}\right)^{2} / 4$. With $(X, Y)=\left(\wp_{2} / \pi^{2}, \wp_{3} / \pi^{3}\right)$, this gives a planar cubic

$$
Y^{2}=X^{2}(X-1)=\left(X^{\prime}\right)^{2} / 4 \subset \mathbb{C P}_{2}(X, Y)
$$

that looks quite similar to (14). Equation (18) should be compared to the Weierstrass cubic in this limit, $\left(X^{\prime}\right)^{2} / 4=X^{3}-\pi^{4} X / 3-2 \pi^{6} / 27$, which is not particularly illuminating.

The similarity between (14) and (18) is to some extent misleading, since we now are dealing with complex functions, and we need to decompress the complex notation in order to draw pictures of what is going on.

With $X=x_{1}+i x_{2}$ and $Y=y_{1}+i y_{2}$, the two slicings discussed in the previous section become

$$
\begin{aligned}
& x_{2}=0=y_{2} \Rightarrow y_{1}^{2}=x_{1}^{3}-x_{1}^{2}, \\
& x_{2}=0=y_{1} \Rightarrow y_{2}^{2}=x_{1}^{2}-x_{1}^{3} .
\end{aligned}
$$

We recognize (19) as the real smooth cubic that we studied above in the section on stereography of circles, so this cycle must be the circle that has not degenerated.

Both cubics are plotted in Figure 11, which also shows the stereographic projection of these cubics onto the $2 \mathrm{D}$ plane $x_{1}=1$, which we can think of as a projection screen. In Figure 11(a), the smooth cubic $y_{1}^{2}=x_{1}^{3}-x_{1}^{2}$ (purple) and the nodal cubic $y_{2}^{2}=x_{1}^{2}-x_{1}^{3}$ (orange) are orthogonal cycles that generate the nodal curve. To see this, we can think of all the endpoints as touching at infinity, represented here by dashed circles intersecting in the point labeled $\infty$. Sliding and shrinking the purple circle along the orange graph, to which it is attached at two points, generates the nodal surface shown in the inset (a pinched torus). Both cycles are projected onto the 2D plane $x_{1}=1$,

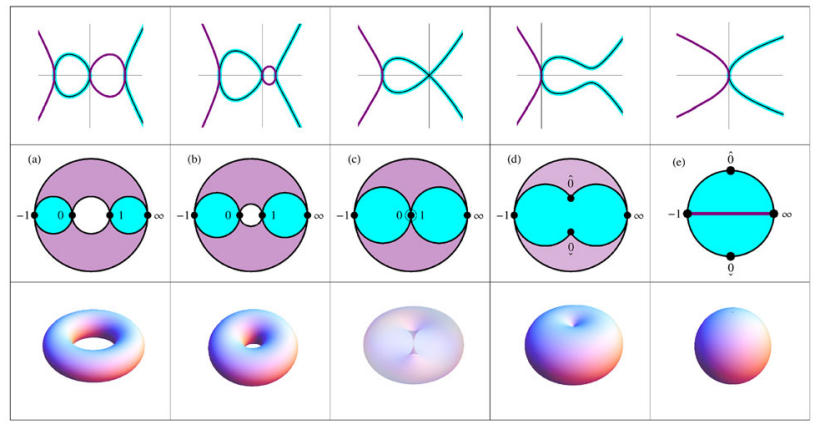

Figure 9. Nodal cubic: shrinking the long cycle.

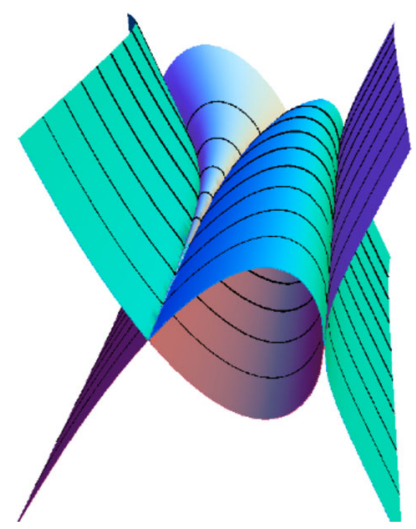

Figure 10. Papillon plot of the stereographic planar cubic in (9), with $\tau=i t, \quad t \in(0.8,2.5), \quad x \in(-1,2), \quad y \in(-1,1) \quad(t$ increases out of the plane of the paper).

which we can think of as the surface of a sphere, by identifying all points at infinity.

Figure 11(b) shows parametric plots of the smooth cubic $\left(1+c_{1}^{2}\right)\left(1, c_{1}\right)$ (purple) parameterized by $c_{1}=\cot \varphi_{1}$, and the nodal cubic $\left(1-c_{2}^{2}\right)\left(1, c_{2}\right)$ (orange) parameterized by $c_{2}=\cot \varphi_{2}$, flattened by drawing both in the same plane. This is the $t \rightarrow \infty$ member of the family whose portrait (papillon plot) is shown in Figure 10. The point $(0,0)$ on the nodal curve projects to $c_{2}= \pm 1$ on the screen. The tail of the node projects to points on the screen outside the interval $c_{2} \in\langle-1,1\rangle$. Unlike the projection of the smooth cubic, which is well defined everywhere, the nodal projection is ill defined at the node and must be blown up at this point. Since the mappings are bijective (except at $(0,0))$, this provides a birational map between the cubic nodal curve and the $2 \mathrm{D}$ screen.

In summary, we have obtained a detailed and consistent picture of the geometry of the nodal cubic. The meromorphic 2D lattice functions labeling the point $\left(\wp_{2}, \wp_{3}\right)$ on a nodal planar cubic are somewhat peculiar complexified stereographic coordinates. This allows us to conclude that the nodal cubic is birational to a sphere.

\section{ACKNOWLEDGMENTS}

The author is grateful for the hospitality of the CERN Theoretical Physics Department (CERN-TH) near Geneva, 


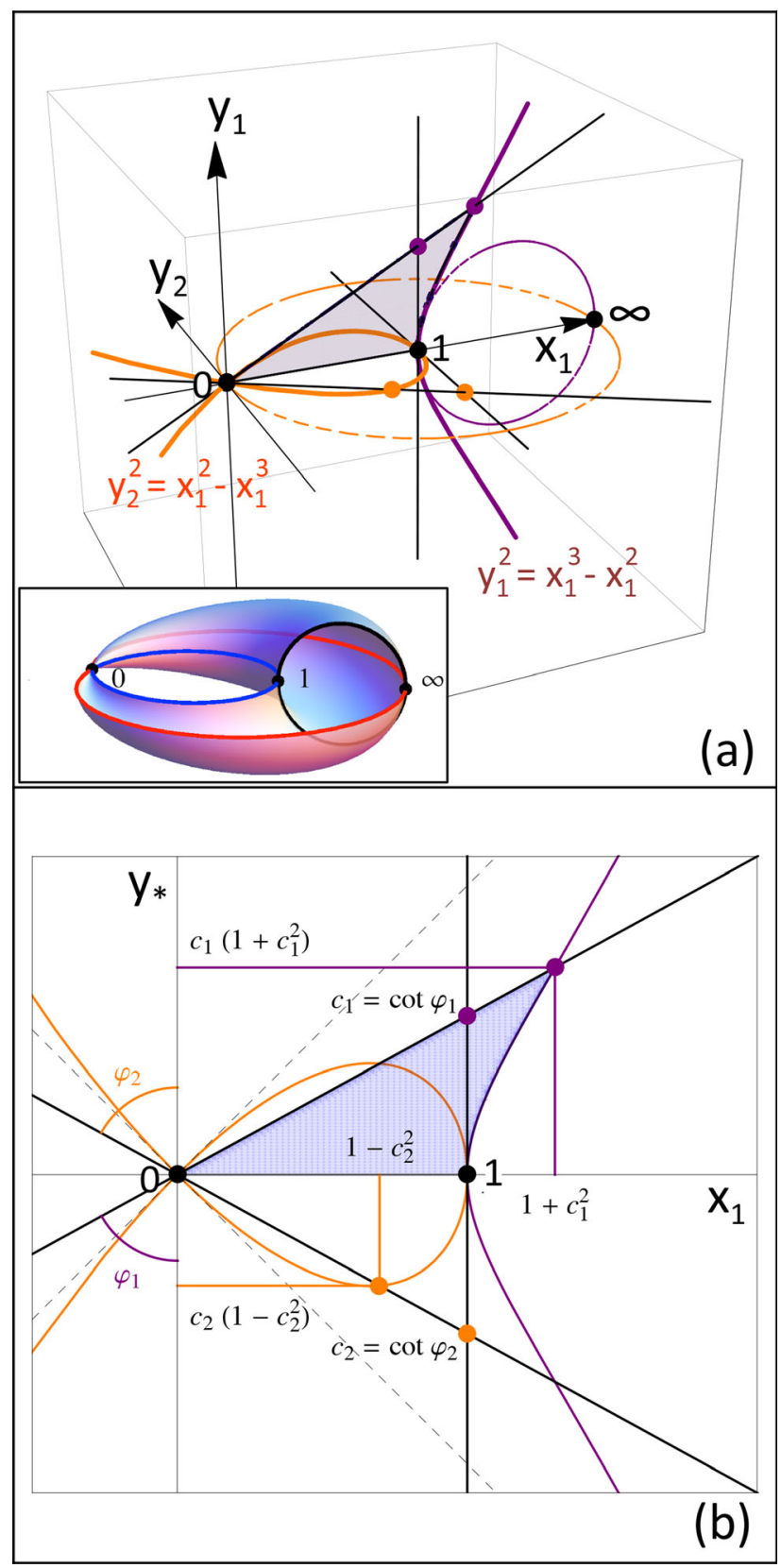

Figure II. Stereography of a complex cubic. (a) The smooth cubic $y_{1}^{2}=x_{1}^{3}-x_{1}^{2}$ (purple) and the nodal cubic $y_{2}^{2}=x_{1}^{2}-x_{1}^{3}$ (orange) are orthogonal cycles that generate the nodal curve. (b) Parametric plots of the smooth cubic (purple) parameterized by $c_{1}=\cot \varphi_{1}$, and the nodal cubic (orange) parameterized by $c_{2}=\cot \varphi_{2}$, have been flattened by drawing both in the same plane.

and the Quantum Computing Technologies Group (QCT) at the Institute for High Energy Physics (IFAE) in Barcelona.

\section{FUNDING}

Open access funding provided by University of Oslo (including Oslo University Hospital).

\section{OPEN ACCESS}

This article is licensed under a Creative Commons Attribution 4.0 International License, which permits use, sharing, adaptation, distribution and reproduction in any medium or format, as long as you give appropriate credit to the original author(s) and the source, provide a link to the Creative Commons licence, and indicate if changes were made. The images or other third party material in this article are included in the article's Creative Commons licence, unless indicated otherwise in a credit line to the material. If material is not included in the article's Creative Commons licence and your intended use is not permitted by statutory regulation or exceeds the permitted use, you will need to obtain permission directly from the copyright holder. To view a copy of this licence, visit http://creativecommons. org/licenses/by/4.0/.

\section{Appendix: Modular Curve Geometry}

Up to normalization, $G_{2}(\tau)$ is a holomorphic connection on the modular curve $\mathcal{X}$. The covariant derivative with this connection generates the Ramanujan identities in (8).

Let $T^{(n-m)}$ denote a mixed tensor with $n$ contravariant (vector) indices and $m$ covariant (covector) indices. Since these indices take only one value on a complex curve, they are usually suppressed. Under a modular coordinate transformation $\tau \rightarrow \tau^{\prime}$, the holomorphic connection $\chi(\tau)=$ $\Gamma_{11}^{1}(\tau)$ and $k$-tensors transform as

$$
\begin{aligned}
\chi(\tau) & \longrightarrow(c \tau+d)^{2} \chi(\tau)+2 c(c \tau+d), \\
T^{( \pm k)} & \longrightarrow(c \tau+d)^{\mp 2 k} T^{( \pm k)},
\end{aligned}
$$

and the covariant derivative with this connection is

$$
\nabla T^{(n-m)}=\left[\frac{\partial}{\partial \tau}+(n-m) \chi\right] T^{(n-m)} .
$$

Holomorphic modular $k$-tensors are called modular forms of weight $w= \pm 2 k$. The ring of all modular forms is generated by $E_{4}$ and $E_{6}$.

The renormalized Eisenstein function $e_{2}(\tau)=\pi i E_{2}(\tau) / 3$ is a holomorphic modular connection, cf. (5), rather than a holomorphic modular tensor (form). Furthermore, since the difference between two connections is a tensor, by subtracting the hyperbolic connection $h_{2}(\tau, \bar{\tau})=i / \mathfrak{I} \tau$ on the upper half-plane, $e_{2}$ can be traded for a quasiholomorphic modular 2-form $\hat{e}_{2}(\tau, \bar{\tau})=e_{2}(\tau)-b_{2}(\tau, \bar{\tau})$.

The only nonvanishing component of the holomorphic modular curvature 2-tensor $(w=4)$ is $\omega=\partial \chi-\chi^{2} / 2$ (with $\partial=\partial / \partial \tau)$, and since the space of forms of weight 4 is onedimensional, $\omega\left(\chi=e_{2}\right)$ must be proportional to $E_{4}$. Matching constant terms gives

$$
\omega=\partial e_{2}-\frac{1}{2} e_{2}^{2}=-\frac{1}{2}\left(\frac{\pi i}{3}\right)^{2} E_{4} .
$$

The covariant derivative of a $k$-tensor produces a $(k+1)$ tensor, i.e., a form of weight $w+2$, from a form of weight $w$. The covariant derivative of $\omega$ is therefore a 3-tensor, and 
since the space of forms of weight 6 is one-dimensional, $\nabla \omega$ must be proportional to $E_{6}$. Matching constant terms, we obtain

$$
\nabla \omega=\left(\partial-2 e_{2}\right) \omega=\left(\frac{\pi i}{3}\right)^{3} E_{6} .
$$

Similarly, the covariant derivative of $\nabla \omega$ produces a 4 tensor, and since the space of forms of weight 8 is onedimensional, $\nabla^{2} \omega$ must be proportional to $E_{4}^{2}$. Matching constants gives

$$
\nabla^{2} \omega=\left(\partial-3 e_{2}\right)\left(\partial-2 e_{2}\right) \omega=-3\left(\frac{\pi i}{3}\right)^{4} E_{4}^{2} .
$$

When these geometric statements (tensor identities on the modular curve), (21)-(23), are rewritten in terms of $E_{2}$ and the derivation $D=\partial / \partial \ln q=(2 \pi i)^{-1} \partial$, we obtain the Ramanujan identities in (8).

C. A. Lütken

Dept. of Physics

University of Oslo

Oslo

Norway

Quantum Research Center

Technology Innovation Institute

Abu Dhabi

United Arab Emirates

e-mail: lutken@fys.uio.no

\section{REFERENCES}

[1] T. M. Apostol. Modular Functions and Dirichlet Series in Number Theory. Springer, 1990.

[2] M. Atiyah. The logarithm of the Dedekind $\eta$-function. Math. Ann. 278 (1987), 335-380.

[3] R. Dedekind. Erläuterungen zu zwei Fragmenten Riemann. In Riemann's Gesammelte Mathematische Werke 2, pp. 466-478. Dover, 1982.

[4] G. Eisenstein. Beiträge zur Theorie der elliptischen Functionen. Journal für die Reine und Angewandte Mathematik 35 (1847), 153-184.

[5] G. H. Hardy. Divergent Series. Clarendon Press, 1949.

[6] E. Hecke. Theorie der Eisensteinschen Reihen höherer Stufe und ihre Anwendung auf Funktionentheorie und Arithmetik. Abh. Math. Sem. Univ. Hamburg 5 (1927), 199-224.

[7] C. A. Lütken. Holomorphic anomaly in the quantum Hall system. Nucl. Phys. B 759 [FS] (2006), 343-369.

[8] C. A. Lütken. Elliptic mirror of the quantum Hall effect. Phys. Rev. B 99:19 (2019), 195152 (31).

[9] S. Ramanujan. On certain arithmetical functions. Trans. Camb. Philos. Soc. 22:9 (1916), 159-184.

[10] A. Weil. Elliptic Functions according to Eisenstein and Kronecker. Springer, 1976.

Publisher's Note Springer Nature remains neutral with regard to jurisdictional claims in published maps and institutional affiliations. 